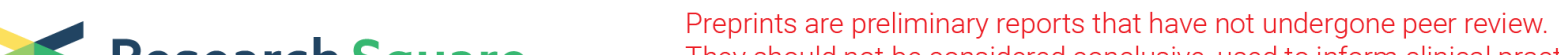 $\begin{array}{ll}\text { Research Square } & \text { They should not be considered conclusive, used to inform clinical practice, } \\ \text { or referenced by the media as validated information. }\end{array}$
}

\section{Retrospective Analysis of Prognostic Factors of Localised or Locally Advanced Prostate Cancer and Their Influence on Treatment Choice}

\section{Sigita Liutkauskiene}

Oncology Institute of Lithuanian University of Health Sciences

Karolina Martinaityte

Medical Academy, Faculty of Medicine, Lithuanian University of Health Sciences

Rasa Malonyte ( $\square$ rasa.malonyte@gmail.com)

Hospital of Lithuanian University of Health Sciences Kaunas Clinics https://orcid.org/0000-0003-42746743

\section{Kristina Jureniene}

Cardiology Institute of Lithuanian University of Health Sciences

\section{Saulius Grizas}

Clinic of Surgery of Lithuanian University of Health Sciences

\section{Elona Juozaityte}

Oncology Institute of Lithuanian University of Health Sciences

\section{Research article}

Keywords: Prostate cancer, prognostic factors, radical prostatectomy, radical radiotherapy

Posted Date: December 7th, 2020

DOl: https://doi.org/10.21203/rs.3.rs-121100/v1

License: (c) (i) This work is licensed under a Creative Commons Attribution 4.0 International License. Read Full License 


\section{Abstract}

Background: According to the majority of retrospective analyses and meta-analyses published in recent years, radical prostatectomy is a more effective treatment method than radical radiotherapy. The objective of this study was to assess whether the treatment method influences the survival of patients with early prostate cancer and to evaluate the impact of patient-dependent and prostate cancerdependent prognostic factors on the choice of radical treatment method of prostate cancer.

Methods: Medical records of selected patients were reviewed. Inclusion criteria involved histologically confirmed prostate adenocarcinoma; localised or locally advanced prostate cancer diagnosed in 20082012; no distant metastases at the time of initial diagnosis; medical records maintain comprehensive data on treatment and follow-up. The influence of prognostic factors on the choice of treatment method was assessed by making comparisons between groups using the Chi-square test.

Results: More favorable prognostic factors were found in the RP treatment group compared to the RT treatment group: patients aged 60 years and younger accounted for $35 \%$ in the RP group, and $18 \%$ - in the RT group, with increasing age the number of RP decreased and the number of RT increased $(p<0.001)$; score of 3-4 points of $\mathrm{CCl}$ was set in 36\% patients in the RP group, and 6\% - in the RT group, with increasing $\mathrm{CCl}$ score, the number of RP decreased and the number of RT increased $(p<0.001)$; baseline PSA level of $10 \mathrm{ng} / \mathrm{ml}$ and less was found in $67 \%$ patients in the RP group, and $52 \%$ - in the RT group, with increasing PSA level, the number of RP decreased and the number of RT increased $(p<0.001)$. Gleason score did not influence the choice of treatment method $(p=0.24)$.

Conclusions: Prognostic factors of localised or locally advanced prostate cancer influenced the choice of treatment method. In case of more favorable prognostic factors, radical prostatectomy was more often chosen.

\section{Background}

Radical prostatectomy and radical radiotherapy are the main treatment options for non-metastatic prostate cancer. Which one of these two methods is more effective still remains the subject of discussion and debate in multidisciplinary team meetings, especially between urologists and radiation oncologists.

According to the majority of retrospective analyses and meta-analyses published in recent years, radical prostatectomy is a more effective treatment method than radical radiotherapy $(1,2,3)$, while radical radiotherapy has been even identified as a factor associated with increased overall mortality in some articles (3). However, international guidelines currently recommend radical prostatectomy and radical radiotherapy as level 1 evidence treatment options without distinguishing any method as more effective $(4,5,6)$. Why do the findings of retrospective meta-analyses differ from international recommendations? In search of an answer to this question, we decided to conduct a retrospective study to analyse the impact of prostate cancer-dependent prognostic factors and patient-dependent prognostic factors on 5- 
year overall survival of localised or locally advanced prostate cancer patients and the influence of these factors on radical treatment choice.

\section{Methods}

\section{Study design and setting}

This retrospective study was approved by the Bioethics Center of Lithuanian University of Health Sciences. Medical records of patients diagnosed with non-metastatic prostate cancer in 2008-2012 in the Affiliate of Hospital of Lithuanian University of Health Sciences Kaunas Clinics Kaunas Oncology Hospital were analysed.

\section{Participants}

Inclusion criteria of the study were the following: 1) histologically confirmed prostate adenocarcinoma; 2) patients treated in the Affiliate of Hospital of Lithuanian University of Health Sciences Kaunas Clinics Kaunas Oncology Hospital; 3) localised or locally advanced prostate cancer diagnosed in 2008-2012;4) no distant metastases at the time of initial diagnosis; 5) medical records maintain comprehensive data on treatment and follow-up. Exclusion criteria were the following: 1) medical records maintain incomplete data on treatment and follow-up; 2) history of other previous malignancies; 3 ) distant metastases suspected at the time of initial diagnosis.

The following data necessary for this study were collected: date of diagnosis, patients age at the time of diagnosis, comorbid conditions, baseline prostate specific antigen (PSA) level, Gleason score, clinical T category (cT) at the time of diagnosis, method of treatment used, pathological T category (pT) after radical prostatectomy, data on the radicality of the operation $(R)$, date of biochemical recurrence, date of occurrence of metastases, date of last visit, date of death. The date of diagnosis was defined as the date when pathology report of histological examination of tumour biopsy was provided. The clinical value of prognostic factors and the effectiveness of the treatment method were assessed by analysing the 5-year overall survival of the patients from the date of histological confirmation of the diagnosis.

Three-dimentional conformal external beam radiotherapy was used for irradiation of prostate cancer patients. Dose-escalated intensity-modulated radiation therapy (IMRT) and image-guided RT were not available at that period in our hospital. Daily fractions of $2 \mathrm{~Gy}$, five fractions per week till 60-70 Gy was standard of care at that time.

The impact of patients' comorbidities on survival was assessed using the age-adjusted Charlson Comorbidity Index (aa-CCl) $(7,8)$. The components of the aa- $\mathrm{CCl}$ and their values are shown in Table 1. 
Table 1

Age-adjusted Charlson Comorbidity Index (aa-CCl)

\begin{tabular}{|l|l|}
\hline Variable & Point \\
\hline Age (years): & 0 \\
\hline$<50-59$ & 1 \\
\hline $60-69$ & 2 \\
\hline $70-79$ & 3 \\
\hline$\geq 80$ & 4 \\
\hline Myocardial infarction & 1 \\
\hline Congestive heart failure & 1 \\
\hline Peripheral vascular disease & 1 \\
\hline Cerebrovascular disease & 1 \\
\hline Dementia & 1 \\
\hline Chronic pulmonary disease & 1 \\
\hline Connective tissue disease & 1 \\
\hline Ulcer disease & 1 \\
\hline Mild liver disease & 2 \\
\hline Diabetes mellitus without end-organ damage & 2 \\
\hline Diabetes mellitus with end-organ damage & 1 \\
\hline Hemiplegia & 2 \\
\hline Renal disease & 2 \\
\hline Any malignancy* & 2 \\
\hline Lymphoma & 2 \\
\hline Leukemia & 2 \\
\hline Moderate or severe liver disease & 2 \\
\hline Metastatic solid tumor** & 2 \\
\hline Acquired immunodeficiency syndrome (AlDS) & 2 \\
\hline$*$ was not counted because all included patients met this & 2 \\
\hline
\end{tabular}




\section{Statistical Methods}

The major goal of the study was to evaluate the influence of prostate cancer-dependent and patientdependent prognostic factors on the choice of radical treatment method. To achieve this goal, we had to assess the impact of prognostic factors and treatment methods on 5-year overall survival. Overall survival at 5 years was defined as being alive 5 years after cancer diagnosis (the date of prostate cancer histological examination), with those alive censored at the last known follow up. The Kaplan-Meier method was used for single variable survival data analysis. Differences of the survival rate were determined using the log-rank test.

The impact of various factors on the 5-year overall survival of prostate cancer patients was assessed using univariate and multivariate Cox's regression analysis. Hazard ratios (HR) and 95\% confidence intervals were calculated.

The influence of prognostic factors on the choice of treatment method was assessed by making comparisons between groups using the Chi-square test.

Level of significance for statistical tests was 0.05 .

Microsoft Office Excel 2007 and Statistical Package for the Social Sciences (SPSS) version 25 were used for data processing and statistical analysis.

\section{Results}

Data of 301 patients who met the inclusion criteria for the study were analysed. For all patients 5-year overall survival was $76.6 \%$. During the 5 -year follow-up period 70 patients $(23.4 \%)$ died and 231 survived for 5 years or more. Distant metastases were diagnosed in 50 patients $(16.6 \%)$ over a 5 -year period.

The process of patients inclusion in the study is shown in Fig. 1.

Characteristics and 5-year overall survival (\%) of the study patients, taking into account prostate cancerdependent and patient-dependent prognostic factors, are presented in Table 2. 
Table 2

Localised or locally advanced prostate cancer patients' characteristics $(N=301)$ and 5-year overall survival

\begin{tabular}{|c|c|c|c|}
\hline Characteristic & $\begin{array}{l}\text { Number of patients } \\
(\mathrm{N})\end{array}$ & $\begin{array}{l}\text { 5-year overall survival } \\
\text { (\%) }\end{array}$ & p-value (log-rank) \\
\hline \multicolumn{4}{|l|}{ Age (years) } \\
\hline$\leq 60$ & 78 & 84.6 & 0.024 \\
\hline $61-65$ & 59 & 79.7 & $\begin{array}{l}\text { Pairwaise } \\
\text { comparisons }\end{array}$ \\
\hline $66-75$ & 128 & 75.0 & $1-4^{\star}, 2-4^{\star}$ \\
\hline$\geq 75$ & 36 & 61.1 & $\begin{array}{l}1-2,1-3,2-3,3-4- \\
n s\end{array}$ \\
\hline \multicolumn{4}{|c|}{$\begin{array}{l}\text { Pathological T category } \\
\text { (pT) }\end{array}$} \\
\hline T1 & 1 & 0 & 0,004 \\
\hline $\mathrm{T} 2$ & 80 & 96.3 & \\
\hline T3 & 78 & 82.1 & \\
\hline T4 & 1 & 0 & \\
\hline \multicolumn{4}{|c|}{ Clinical T category (cT) } \\
\hline T1 & 1 & 100 & 0.124 \\
\hline T2 & 26 & 80.8 & \\
\hline T3 & 114 & 59.6 & \\
\hline \multicolumn{4}{|c|}{ Baseline PSA (ng/ml) } \\
\hline$<10$ & 170 & 84.1 & $<0.001$ \\
\hline $10-20$ & 72 & 77.8 & $\begin{array}{l}\text { Pairwaise } \\
\text { comparisons }\end{array}$ \\
\hline$\geq 20$ & 59 & 54.2 & $1-2 \mathrm{~ns}, 1-3^{\star \star}, 2-3^{*}$ \\
\hline \multicolumn{4}{|c|}{ Gleason score (points) } \\
\hline $3-6$ & 218 & 82.6 & $<0.001$ \\
\hline 7 & 46 & 71.7 & $\begin{array}{l}\text { Pairwaise } \\
\text { comparisons }\end{array}$ \\
\hline $8-10$ & 37 & 48.6 & $1-2 \mathrm{~ns}, 1-3^{\star *}, 2-3^{*}$ \\
\hline
\end{tabular}

pairwise comparisons ${ }^{*} \mathrm{p}<.05,{ }^{*} \mathrm{p}<.001, \mathrm{~ns}-$ not significant 


\begin{tabular}{|c|c|c|c|}
\hline Characteristic & $\begin{array}{l}\text { Number of patients } \\
(\mathrm{N})\end{array}$ & $\begin{array}{l}\text { 5-year overall survival } \\
\text { (\%) }\end{array}$ & p-value (log-rank) \\
\hline \multicolumn{4}{|c|}{ Score of aa-CCl (points) } \\
\hline $3-4$ & 54 & 100 & $<0.05$ \\
\hline $5-6$ & 90 & 88.9 & $\begin{array}{l}\text { Pairwaise } \\
\text { comparisons }\end{array}$ \\
\hline $7-8$ & 74 & 67.6 & $1-2^{\star}, 1-3^{\star \star}, 1-4^{\star \star}$ \\
\hline$\geq 9$ & 83 & 56.6 & $2-3^{\star \star}, 2-4^{\star \star}, 3-4 \mathrm{~ns}$ \\
\hline
\end{tabular}

Using Kaplan-Meier method, we found that 5-year overall survival in patients with localised or locally advanced prostate cancer is impacted by patient age, pathological T category (pT), baseline PSA level, Gleason score and the score of age-adjusted Charlson Comorbidity Index (Table 2)

\section{Analysis of the impact of patient-dependent prognostic factors on 5-year overall survival}

Kaplan-Meier method was used to analyse the impact of patients age and comorbidities on 5-year overall survival.

It was proved that the patients age at the time of diagnosis had an impact on the 5-year overall survival $(p=0.024)$. Comparing survival among age groups, patients aged 60 years and younger had a higher $5-$ year overall survival rate compared with patients aged 75 years and older $(p=0.003)$ and patients aged 61-65 years had a higher 5-year overall survival rate compared with patients aged 75 years and older ( $p$ $=0.046$ ) as well. No impact of age on survival was found in the other age groups (Table 2).

Patients with a higher score of aa-CCl had a significant deterioration in 5 -year overall survival $(p<0.001)$.

There was no significant difference in survival rates between patients with aa-CCI score of 7-8 points compared with patients with aa-CCl score of 9 points or more $(p=0.113)$ (Table 2$)$.

\section{Analysis of the impact of prostate cancer-dependent prognostic factors on 5-year overall survival}

Kaplan-Meier method was used to analyse the impact of pathological T category (pT), baseline PSA level and Gleason score on 5-year overall survival.

Radical prostatectomy (RP) was performed in 161 patients (53.4\%) and pathological T category (pT) was evaluated for these patients. There was statistically significant higher survival in patients with stage pT2 disease compared with patients with stage pT3 disease $(p=0.004)$. 31 patients $(19,37 \%)$ had not radical (R1) prostatectomy (27 patients had stage pT3 disease and 4 patients had stage pT2 disease). 28 patients $(90,3 \%)$ who had not radical surgery, received postoperative external radiation therapy. Radical 
prostatectomy with lymphonodectomy was performed in 45 patients $(28,12 \%)$ of whom 32 patients had stage $\mathrm{PT} 3$ disease and 13 patients had stage PT2 disease.

External radical radiotherapy (RT) was performed in 103 patients (34.3\%). 31 patients (10.3\%) were treated with hormone therapy alone. Clinical T category (cT) was assessed in patients treated with external radical radiotherapy and hormone therapy. The most commonly $(n=114 ; 81 \%)$ patients were diagnosed with stage cT3 prostate cancer. There was no statistically significant impact of cT on 5-year overall survival $(p=0.124)$.

\section{Analysis of the impact of prognostic factors and treatment method on 5-year overall survival}

5-year overall survival of patients treated with radical prostatectomy was $87 \%$ and $70,9 \%$ of patients treated with radical radiotherapy $(p=0.002)$. Based on this result, RP appears to lead to more favorable patients outcomes and prognosis. This result is consistent with the results of previous meta-analyses ( 1 , $2,3)$.

To identify all factors that determine the survival of patients with localised or locally advanced prostate cancer, we conducted an univariate and multivariate Cox regression analysis to assess the impact of patient-dependent and prostate-cancer-dependent prognostic factors along with treatment method on survival.

Using Cox regression analysis model, we analysed the impact of patients age, baseline PSA, comorbidities, Gleason score, pathological T category and treatment method on 5-year overall survival. Multivariate Cox regression analysis showed 2.54 times higher death risk over 5 years in patients with Gleason score of 8-10 (HR $=2.54,95 \% \mathrm{Cl}: 1.395-4.632, \mathrm{p}=0.002)$. Each point of age-adjusted Charlson Comorbidity Index was found to increase the risk of death 1.21-fold over a 5 -year period $(H R=1.21,95 \%$ Cl: $1.107-1.328, p<0.001)$.

Univariate analysis showed that patients treated with radical RT had 2.34 times higher death risk $(\mathrm{p}=$ $0.003)$ and patients treated with hormone therapy alone had 3.13 times higher death risk $(p<0.001)$ compared with patients for whom an operation was performed. Multivariate analysis did not demonstrate a negative impact of RT on survival compared to RP $(p=0.286)$, but patients who received hormone therapy alone had 2.49 times higher death risk than patients who underwent surgery $(p=0.023)$. Table 3 . 
Table 3

Univariate and multivariate Cox regression analysis of the impact of prognostic factors and treatment method on survival

\begin{tabular}{|lllllll|}
\hline Variables & \multicolumn{3}{l}{ Univariate model } & \multicolumn{4}{c|}{ Multivariate model } \\
\cline { 2 - 7 } & HR & $\mathbf{9 5 \%} \mathbf{C l}$ & p-value & HR & $95 \%$ Cl & p-value \\
\hline Age & 1.04 & $1.013-1.075$ & 0.005 & 0.99 & $0.961-1.021$ & 0.553 \\
\hline Baseline PSA level & 1.01 & $1.005-1.015$ & $<0.001$ & 1.00 & $0.994-1.007$ & 0.970 \\
\hline Pathological T category (pT) & 2.77 & $1.588-4.848$ & $<0.001$ & 1.31 & $0.671-2.558$ & 0.430 \\
\hline Gleason score & 1 & $0.923-3.255$ & 0.087 & 1 & $0.656-2.422$ & 0.488 \\
3-6 points & 1.73 & $2.256-6.811$ & $<0.001$ & 1.26 & $1.395-4.632$ & 0.002 \\
7 points & 3.92 & & & 2.54 & & \\
8-10 points & & & & & & \\
\hline Score of aa-CCl & 1.30 & $1.204-1,404$ & $<0.001$ & 1.21 & $1.107-1.328$ & $<0.001$ \\
\hline Treatment method & 1 & $1.337-4.080$ & 0.003 & 1 & $0.750-2.654$ & 0.286 \\
Prostatectomy & 2.34 & $3.128-11.049$ & $<0.001$ & 1.41 & $1.134-5.449$ & 0.023 \\
Radiotherapy & 3.13 & & & 2.49 & & \\
Hormone therapy & & & & & & \\
\hline
\end{tabular}

Analysis of the influence of prognostic factors on the choice of radical treatment method

In order to find out the criteria that determine the choice of treatment option in daily clinical practice, we analysed the influence of patient-dependent prognostic factors (age, the score of age-adjusted Charlson Comorbidity Index) and prostate cancer-dependent prognostic factors (Gleason score, baseline PSA) on treatment choice.

We found that RP was more common in patients with better prognostic 5-year overall survival according to Kaplan Meier method, it means that RP was statistically significant chosen in patients younger than 60 years old $(p<0.001)$, with a lower score of age-adjusted Charlson Comorbidity Index $(p<0.001)$ and with lower baseline PSA level $(p<0.001)$ (Fig. 2).

More favorable prognostic factors were found in the RP treatment group compared to the RT treatment group: patients aged 60 years and younger accounted for 35\% in the RP group, and 18\% - in the RT group, with increasing age the number of RP decreased and the number of RT increased ( $p<0.001)$; score of 34 points of aa-CCl was set in $36 \%$ patients in the RP group, and 6\% - in the RT group, with increasing aa$\mathrm{CCl}$ score, the number of RP decreased and the number of RT increased $(p<0.001)$; baseline PSA level of $10 \mathrm{ng} / \mathrm{ml}$ and less was found in $67 \%$ patients in the RP group, and $52 \%$ - in the RT group, with increasing PSA level, the number of RP decreased and the number of RT increased $(p<0.001)$ (Fig. 2). 
Gleason score did not influence the choice of treatment method $(p=0.24)$.

\section{Discussion}

According to many retrospective meta-analyses, radical prostatectomy results in better overall survival rates compared to radical radiotherapy $(1,2,3,4)$. The findings of the studies were presented as undisputed and recommended prioritising RP for all patients with localised or locally advanced prostate cancer. However, these meta-analyses have their limitations. For example, Walis et al. did not analyse the impact of patient-dependent and prostate cancer-dependent prognostic factors on the survival rates and the influence of these factors on the choice of treatment method. In the study of Sooriakumaran et al. it was analysed the impact of RP and RT on survival according to patients' risk groups, but there was no analysis of patient-dependent risk factors as well as no analysis of the influence of prognostic factors on treatment choice. It was clearly demonstrated that the two patients' groups (treated with RP or RT) in this study are definitely different. In our study it was shown that almost all clinico-pathological data significantly favored surgery. In fact, most of the patients treated with RT were older, with more comorbidities and worse features of prostate cancer (eg, higher Gleason score and higher baseline PSA levels) than surgically treated patients.

In our study using a multivariate Cox regression analysis model, we found that Gleason score and patients' comorbidities had a significant impact on 5-year overall survival in localised or locally advanced prostate cancer patients. Patients who received hormone therapy alone had 2.49 times higher death risk compared with patients who underwent radical surgery. Can we say that hormone therapy increases the risk of death compared to surgical treatment? Definitely not, as evidenced by the data presented in Fig. 2, which reveals that hormone therapy alone was given to patients with aa-CCl score of 7 or more, $64 \%$ patients had baseline PSA greater than $20 \mathrm{ng} / \mathrm{ml}, 80 \%$ patients were 66 years and older - that is, hormone therapy was given to patients with poorer prognosis.

In our study, patients treated with RP and RT had a 5-year overall survival of $87 \%$ and $70.9 \%$, respectively $(p=0.002)$. However, after analysing the distribution of prognostic factors in the treatment groups, we found that patients with better prognosis (younger than 60 years $(p<0.001)$, with a lower score of aa-CCl $(p<0.001)$ and lower baseline PSA $(p<0.001)$ were more likely to be treated with RP (Fig. 2$)$.

Therefore, based on the data of our study, we conclude that it is not the local treatment method that affects survival in prostate cancer patients, but the patient-dependent and disease-dependent prognostic factors which influence the choice of treatment method.

Another important aspect we would like to highlight is the need for RT after RP. RT after RP is commonly used after non-radical (R1) surgery (about $20 \%$ of cases in our study) and after the development of biochemical recurrence (biochemical recurrence after RP occurred in $27 \%$ patients and after RT - in $8.7 \%$ patients). How should we assess this fact? Should we just know that for about $45 \%$ of patients RT will be necessary after RP? Or perhaps the combination of these two treatment methods improves patients' prognosis and survival rates, as it was found in the study by Fahmy et al. (9)? There are many examples 
in oncology where a combination of these two treatment methods results in better treatment outcomes (breast cancer, colorectal cancer, etc.).

Could prospective randomised clinical trials today answer the question which one of these two treatment methods is more effective? There is no doubt that this is the only way to evaluate the possible superiority of one treatment method over another, but it requires new radiological technologies, experienced physicians, accurate methods to assess the stage of prostate cancer, molecular markers, as prostate cancer is a very heterogeneous disease with a very heterogeneous patients' population $(10,11)$.

\section{Conclusions}

Results of our study showed that the survival of localised or locally advanced prostate cancer patients was determined by patient-dependent and disease-dependent prognostic factors which definitely influenced the choice of treatment method. Today it becomes clear that the impact of treatment method on survival in retrospective studies can't be assessed due to the significant heterogeneity of patients with localised or locally advanced prostate cancer.

\section{Abbreviations}

aa-CCl

Age-adjusted Charlson Comorbidity Index

HR

Hazard ratio

RP

Radical prostatectomy

RT

Radical radiotherapy

PSA

Prostate specific antigen

Gy

Gray

HT

Hormone therapy

\section{Declarations}

\section{Ethics approval and consent to participate}

Study was approved by the Bioethics Center of Lithuanian University of Health

\section{Consent for publication}


Not applicable

\section{Availability of data and materials}

The data analysed during the current study are available from the corresponding author on reasonable request.

\section{Competing interests}

The authors declare that they have no competing interests.

\section{Funding}

This study received no specific grant from any funding agency in the public, commercial, or not-for-profit sectors.

\section{Authors' contributions}

SL conceived of the study, participated in its coordination and wrote the manuscript; EJ, SG and RM participated in the study conception, data interpretation, developing and writing of the manuscript. KJ performed statistical analysis. KM selected cases, reviewed medical records. All authors read and approved the final manuscript.

\section{Acknowledgements}

We greatly appreciate all the authors for their endeavor.

\section{References}

1. Wallis CJD, Saskin R, Choo R, Herschorn S, Kodama RT, Satkunasivam R, et al. Surgery Versus Radiotherapy for Clinically-localized Prostate Cancer: A Systematic Review and Meta-analysis. Eur Urol. 2016 Jul;70(1):21-30. doi: 10.1016/j.eururo.2015.11.010. Epub 2015 Dec 15. PMID: 26700655.

2. Sooriakumaran P, Nyberg T, Akre O, Haendler L, Heus I, Olsson M, et al. Comparative effectiveness of radical prostatectomy and radiotherapy in prostate cancer: observational study of mortality outcomes. BMJ. 2014 Feb;26;348:g1502. doi:10.1136/bmj.g1502. PMID: 24574496; PMCID: PMC3936107.

3. Nepple KG, Stephenson AJ, Kallogjeri D, Michalski J, Grubb RL 3rd, Strope SA, et al. Mortality after prostate cancer treatment with radical prostatectomy, external-beam radiation therapy, or brachytherapy in men without comorbidity. Eur Urol. 2013 Sep;64(3):372-8. doi:10.1016/j.eururo.2013.03.005. Epub 2013 Mar 13. PMID: 23506834; PMCID: PMC3930076.

4. Petrelli F, Vavassori I, Coinu A, Borgonovo K, Sarti E, Barni S. Radical prostatectomy or radiotherapy in high-risk prostate cancer: a systematic review and metaanalysis. Clin Genitourin Cancer. 2014 Aug;12(4):215-24. doi:10.1016/j.clgc.2014.01.010. Epub 2014 Feb 6. PMID: 24589471. 
5. Bill-Axelson A, Holmberg L, Garmo H, Rider JR, Taari K, Busch C, et al. Radical prostatectomy or watchful waiting in early prostate cancer. N Engl J Med. 2014 Mar 6;370(10):932 - 42. doi: 10.1056/NEJMoa1311593. PMID: 24597866; PMCID: PMC4118145.

6. Wilt TJ, Brawer MK, Jones KM, Barry MJ, Aronson WJ, Fox S, et al. Prostate Cancer Intervention versus Observation Trial (PIVOT) Study Group. Radical prostatectomy versus observation for localized prostate cancer. N Engl J Med. 2012 Jul 19;367(3):203 - 13. doi:

10.1056/NEJMoa1113162. Erratum in: N Engl J Med. 2012 Aug 9;367(6):582. PMID: 22808955; PMCID: PMC3429335.

7. Charlson M, Szatrowski TP, Peterson J, Gold J. Validation of a combined comorbidity index. J Clin Epidemiol. 1994 Nov;47(11):1245-51. doi: 10.1016/0895-4356(94)90129-5. PMID: 7722560.

8. Chang CM, Yin WY, Wei CK, Wu CC, Su YC, Yu CH. et al. Adjusted Age-Adjusted Charlson Comorbidity Index Score as a Risk Measure of Perioperative Mortality before Cancer Surgery. PLoS One. 2016 Feb 5;11(2):e0148076. doi: 10.1371/journal.pone.0148076. Erratum in: PLoS One. 2016;11(6):e0157900. PMID: 26848761; PMCID: PMC4744039.

9. Fahmy O, Khairul-Asri MG, Hadi SHSM, Gakis G, Stenzl A. The Role of Radical Prostatectomy and Radiotherapy in Treatment of Locally Advanced Prostate Cancer: A Systematic Review and MetaAnalysis. Urol Int. 2017;99(3):249-256. doi: 10.1159/000478789. Epub 2017 Jul 4. PMID: 28675891.

10. Roach M 3rd. Radical prostatectomy $\mathrm{v}$ radiation: only a randomised trial can provide the answer. BMJ. 2014 Mar 25;348:g2266. doi: 10.1136/bmj.g2266. PMID: 24668796.

11. Spahn M, Dal Pra A, Aebersold D, Tombal B. Radiation Therapy Versus Radical Prostatectomy: A Never-ending Discussion. Eur Urol. 2016 Jul;70(1):31-2. doi:10.1016/j.eururo.2016.01.049. Epub 2016 Feb 5. PMID: 26856959.

\section{Figures}




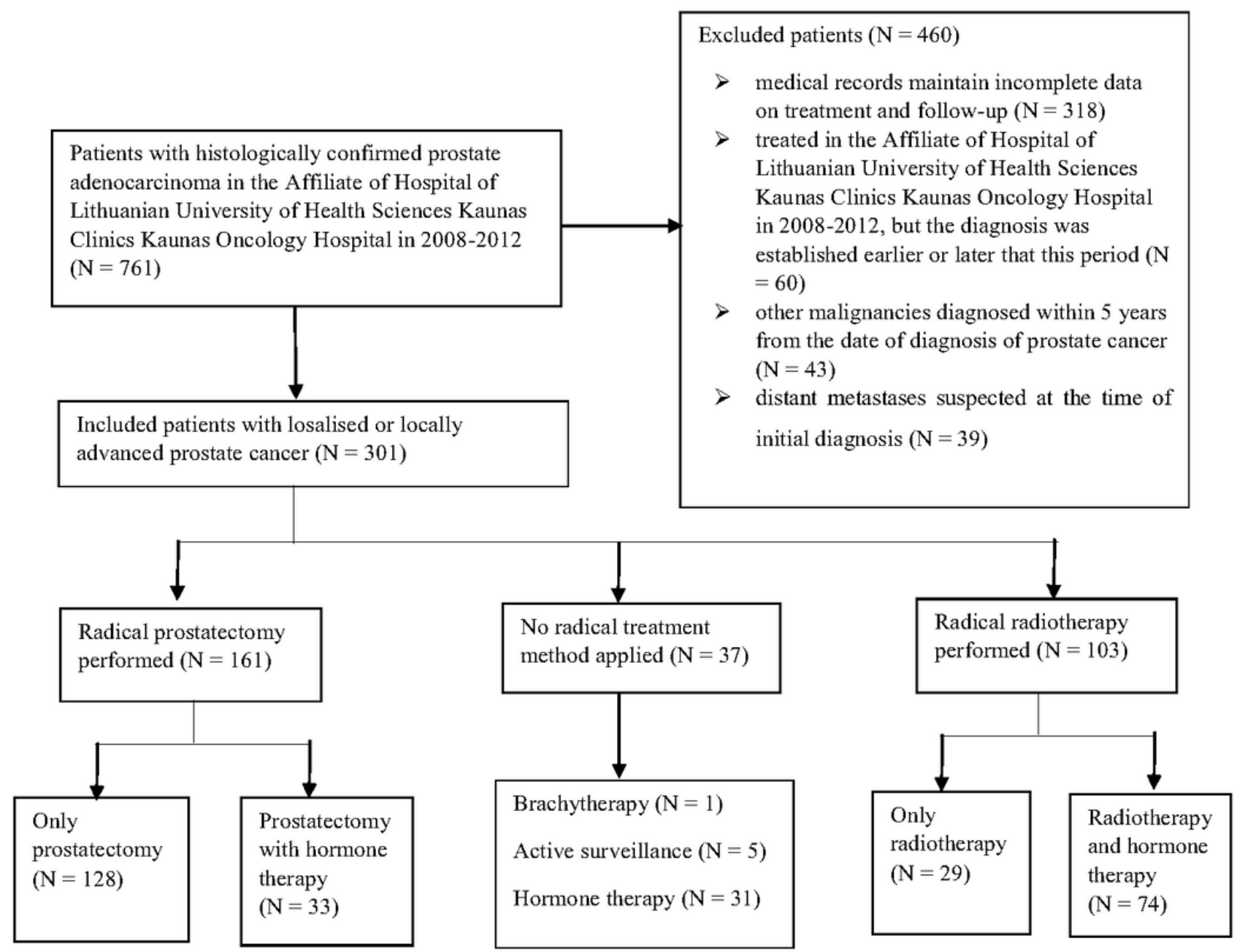

\section{Figure 1}

Flow chart of cases used for analysis 

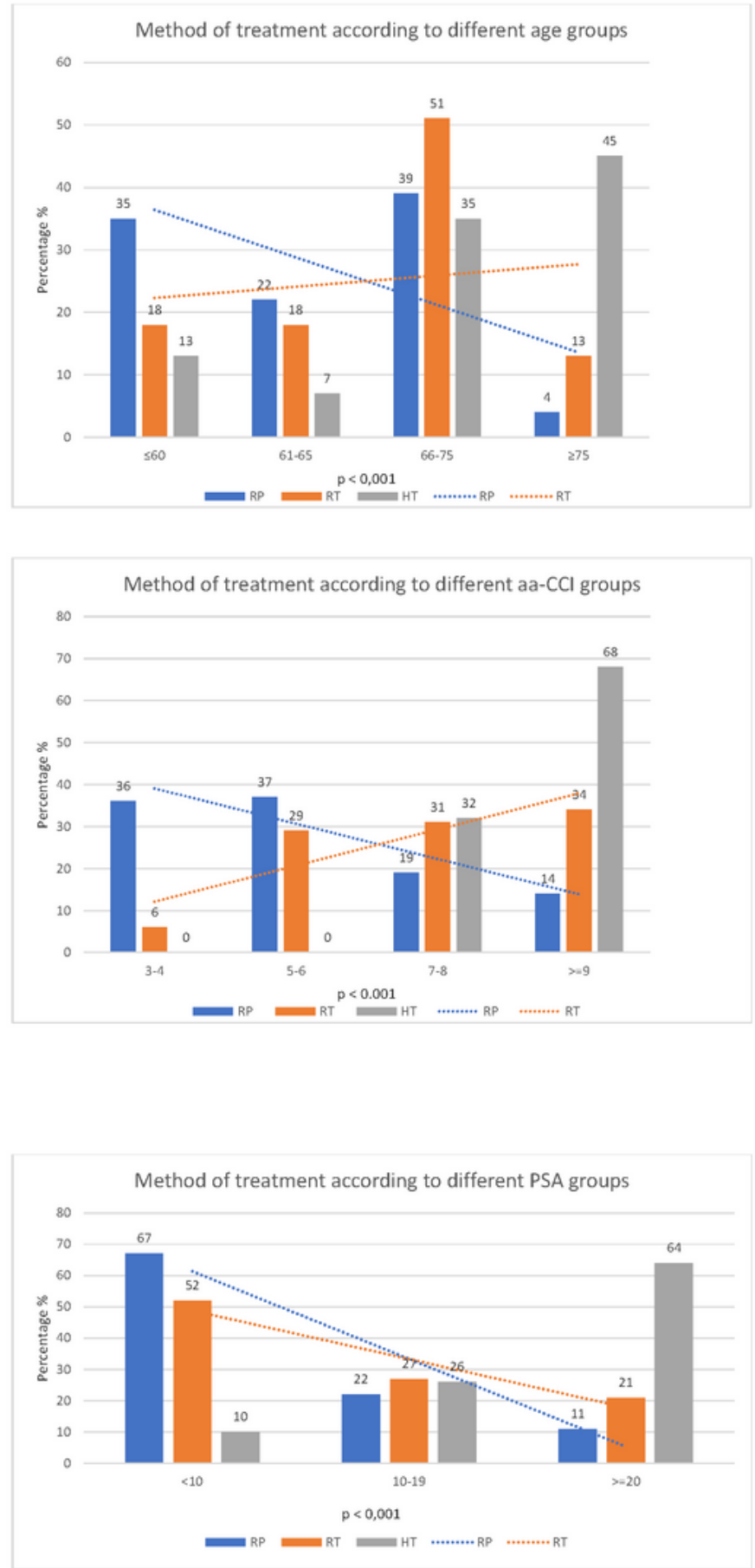

Figure 2

Analysis of prognostic factors influencing treatment choice 